2 Examination of two-dimensional gas chromatography with a nitrogen 3 chemiluminescence detector to facilitate quantitation and 4 characterization of nitrogen-containing compounds in petroleum5 derived fuels

Mark Romanczyk, ${ }^{*}, a, b$ Rachel D. Deese, ${ }^{c, d}$ Thomas N. Loegel, ${ }^{c}$ Alison E. Metz, ${ }^{a}$ Robert E. Morris, ${ }^{e}$ Richard A. Kamin, ${ }^{a}$ Andrew M. McDaniel, ${ }^{a}$ and 9 Michael E. Pereticha

a Naval Air Systems Command, 22229 Elmer Road, Bldg 2360, Patuxent River, Maryland 20670, United States

b Peraton, 12975 Worldgate Drive, Herndon, Virginia 20170-6008, United States

c US Naval Research Laboratory, 4555 Overlook Avenue S.W., Washington, District of Columbia 20375, United States

d NRC Research Associateships Programs, 500 Fifth Street, Washington, District of

e Nova Research, Inc., 1900 Elkin Street, Suite 230, Alexandria, Virginia 22308, United States

\title{
Nitrogen-containing compounds.
}

7-Azaindole (98\% purity), benzothiazole (97\% purity), 2,3-dimethylindole (97\% purity), o-anisidine (99\% purity), octadecylamine (> 99\% purity), 3-methylindole (99\% purity), were purchased from Alfa Aesar. N,N-dimethyldodecylamine (97\%) was purchased from TCl. Pyridine anhydrous (99.8\% purity), pyrrole (98\% purity), 2,4,6-trimethylpyridine (99\% purity), N,N-diethylaniline ( $\geq$ $99 \%$ purity), 1-butylimidazole (98\% purity), quinoline (98 \% purity), carbazole (> 95\% purity), $N, N-$ dimethylhexylamine (95\% purity), 2-aminoheptane (99\% purity), N,N-dimethyloctylamine (> 95\%), $N, N$-dimethyldecylamine (99\% purity), $N$-methyldodecylamine (97\% purity), 2-aminooctane Sigma Aldrich (99\% purity), 3-methylquinoline (> 99\%) N,N-dimethyltetradecylamine $(95 \%$ purity), tetradecylamine (95\% purity), 2,5-dimethylpyrrole (98\% purity), N-methylaniline (98\% purity), 4-ethylaniline (98\% purity), 1,2,3,4-tetrahydroquinoline (98\% purity), acridine (97\% purity), phenothiazine ( $>98$ purity), indole ( $\geq 99 \%$ purity), 9-ethylcarbazole (97\% purity), aniline $(\geq 99.5 \%$ purity), 2-propylaniline (97\% purity) 2,5-dimethylindole (97\% purity), 4-tert-butylaniline (99\% purity), 1-methylimidazole (99\% purity), 2,2'-biquinoline (98\% purity) and $11 \mathrm{H}$-benzo[a]carbazole ( $100 \%$ purity) were purchased from Sigma Aldrich. 
Table S1. The y-intercepts and slopes for three different trend lines of carbazole prepared on three separate days measured a week apart from each other. The LOD and LOQ of nitrogen for the different trend lines are provided.

\begin{tabular}{|c|c|c|c|}
\hline \multirow[t]{2}{*}{ Measurement } & Non-weighted & Weighted $(1 / x)$ & Weighted $\left(1 / x^{2}\right)$ \\
\hline & \multicolumn{3}{|c|}{$\mathrm{Y}$ - intercept values } \\
\hline 1 & $-111,307,851.89$ & $-7,699,443.58$ & $-3,615,316.60$ \\
\hline 2 & $-103,820,232.46$ & $-9,479,093.90$ & $-3,728,938.36$ \\
\hline 3 & $-85,259,898.17$ & $-8,899,945.98$ & $-3,386,281.30$ \\
\hline \multirow[t]{2}{*}{ Standard deviation } & $13,410,483.13$ & 907723.67 & 174537.93 \\
\hline & \multicolumn{3}{|c|}{ Values for the slope of trendline } \\
\hline 1 & $500,352,583.92$ & $487,935,984.48$ & $437,664,053.98$ \\
\hline 2 & $456,520,926.44$ & $445,239,102.68$ & $378,114,620.36$ \\
\hline 3 & $491,169,957.17$ & $481,903,471.11$ & $431,548,170.62$ \\
\hline Average & $482,681,155.84$ & $471,692,852.76$ & $415,775,614.99$ \\
\hline LOD (ppm) & 0.083 & 0.006 & 0.001 \\
\hline LOQ (ppm) & 0.278 & 0.020 & 0.004 \\
\hline
\end{tabular}

40

41

42

43

44

45

46

47

48

49

50

6

0

Table S2. The linear trend lines for the NCCs plotted in Figure 1 are shown. Carbazole (1) and (2) refer to equimolar mixtures 1 and 2, respectively.

\begin{tabular}{|c|c|c|}
\hline \multicolumn{2}{|c|}{ Equimolar solution 1 } & \\
\hline Analyte & Linear formula & $R^{2}$ value \\
\hline Aniline & $\mathrm{y}=464,325,553.1 \mathrm{x}-67,682,680.4$ & 0.9998 \\
\hline Quinoline & $\mathrm{y}=499,432,104.1 \mathrm{x}-42,726,919.4$ & 1.0000 \\
\hline $\mathrm{N}, N$-dimethyloctylamine & $\mathrm{y}=493,969,753.8 \mathrm{x}-113,745,800.5$ & 0.9997 \\
\hline Carbazole $^{1}$ & $\mathrm{y}=512,395,249.7 \mathrm{x}-108,457,515.2$ & 0.9996 \\
\hline $\mathrm{N}, N$-dimethyldodecylamine & $\mathrm{y}=523,837,021.1 \mathrm{x}-137,012,824.8$ & 0.9995 \\
\hline \multicolumn{1}{|c|}{ Equimolar solutions 2 and 3 } & $R^{2}$ value \\
\hline Analyte & $\mathrm{Linear}$ formula & 0.9998 \\
\hline Pyrrole & $\mathrm{y}=363,276,410.9 \mathrm{x}-88,862,441.2$ & 0.9998 \\
\hline Pyridine & $\mathrm{y}=518,503,218.2 \mathrm{x}-107,318,030.5$ & 0.9992 \\
\hline
\end{tabular}

7

8

9


Table S4. The slopes of five trend lines of carbazole over the course of five weeks. The average value of all slopes and standard and relative standard deviations are provided.

\begin{tabular}{|c|c|}
\hline Carbazole & Slope from trendline \\
\hline \multirow{3}{*}{ Slope values } & 479333270.6 \\
\cline { 2 - 2 } & 541274514.1 \\
\cline { 2 - 2 } & 504578837.6 \\
\cline { 2 - 2 } & 547595284.9 \\
\cline { 2 - 2 } & 497113910.2 \\
\hline Average of slope values & 511708066.9 \\
\hline Standard deviation & 26843833.43 \\
\hline Relative standard deviation & $5.25 \%$ \\
\hline
\end{tabular}

Table S3. The averages and standard deviations for blob volumes of nitrogen in molecules aniline, quinoline and carbazole produced by the GCxGC NCD from three separate measurements a week apart from each other by using freshly prepared standards.

\begin{tabular}{|l|l|l|l|l|l|c|}
\hline \multirow{2}{*}{$\mathrm{ppm}$} & \multicolumn{2}{|c|}{ Aniline } & \multicolumn{2}{c|}{ Quinoline } & \multicolumn{2}{c|}{ Carbazole (1) } \\
\cline { 2 - 7 } & Average & \multicolumn{1}{c|}{ SD } & Average & \multicolumn{1}{c|}{ SD } & Average & SD \\
\hline 0.020 & $4.55 \mathrm{E} 6$ & $2.29 \mathrm{E} 5$ & $6.08 \mathrm{E} 6$ & $8.33 \mathrm{E} 5$ & $3.94 \mathrm{E} 6$ & $3.36 \mathrm{E} 5$ \\
\hline 0.025 & $5.96 \mathrm{E} 6$ & $5.62 \mathrm{E} 5$ & $7.51 \mathrm{E} 6$ & $8.45 \mathrm{E} 5$ & $5.80 \mathrm{E} 6$ & $6.64 \mathrm{E} 5$ \\
\hline 0.050 & $1.48 \mathrm{E} 7$ & $4.40 \mathrm{E} 5$ & $1.65 \mathrm{E} 7$ & $2.34 \mathrm{E} 5$ & $1.27 \mathrm{E} 7$ & $9.41 \mathrm{E} 5$ \\
\hline 0.100 & $3.26 \mathrm{E} 7$ & $1.32 \mathrm{E} 6$ & $3.45 \mathrm{E} 7$ & $3.05 \mathrm{E} 6$ & $2.82 \mathrm{E} 7$ & $2.48 \mathrm{E} 6$ \\
\hline 0.250 & $8.77 \mathrm{E} 7$ & $9.92 \mathrm{E} 5$ & $9.80 \mathrm{E} 7$ & $4.88 \mathrm{E} 6$ & $8.44 \mathrm{E} 7$ & $4.04 \mathrm{E} 6$ \\
\hline 0.500 & $1.93 \mathrm{E} 8$ & $7.84 \mathrm{E} 6$ & $2.09 \mathrm{E} 8$ & $2.40 \mathrm{E} 7$ & $1.86 \mathrm{E} 8$ & $6.86 \mathrm{E} 6$ \\
\hline 1.00 & $4.15 \mathrm{E} 8$ & $9.26 \mathrm{E} 6$ & $4.56 \mathrm{E} 8$ & $3.52 \mathrm{E} 7$ & $4.10 \mathrm{E} 8$ & $1.73 \mathrm{E} 7$ \\
\hline 5.00 & $2.16 \mathrm{E} 9$ & $6.20 \mathrm{E} 7$ & $2.38 \mathrm{E} 9$ & $1.46 \mathrm{E} 8$ & $2.28 \mathrm{E} 9$ & $1.39 \mathrm{E} 8$ \\
\hline 10.0 & $4.39 \mathrm{E} 9$ & $7.22 \mathrm{E} 7$ & $4.88 \mathrm{E} 9$ & $2.89 \mathrm{E} 8$ & $4.77 \mathrm{E} 9$ & $1.66 \mathrm{E} 8$ \\
\hline 25.0 & $1.14 \mathrm{E} 10$ & $2.39 \mathrm{E} 8$ & $1.24 \mathrm{E} 10$ & $5.96 \mathrm{E} 8$ & $1.24 \mathrm{E} 10$ & $4.12 \mathrm{E} 8$ \\
\hline 50.0 & $2.33 \mathrm{E} 10$ & $9.84 \mathrm{E} 8$ & $2.50 \mathrm{E} 10$ & $1.13 \mathrm{E} 9$ & $2.57 \mathrm{E} 10$ & $1.39 \mathrm{E} 9$ \\
\hline
\end{tabular}

(1)

6

9

(1)

1

2 Table S5. The trend lines and $\mathrm{R}^{2}$ values for analytes with additional heteroatom.

\begin{tabular}{|c|c|c|}
\hline \multicolumn{3}{|c|}{ Heteroatom-containing compounds } \\
\hline Analyte & Linear formula & $R^{2}$ \\
\hline Carbazole & $\mathrm{y}=594,483,997.03 \mathrm{x}-90,547,712.46$ & 0.9998 \\
\hline o-Anisidine & $\mathrm{y}=589,250,190.00 \mathrm{x}+11,657,458.25$ & 1.000 \\
\hline Benzothiazole & $\mathrm{y}=577,835,369.16 \mathrm{x}+54,268,135.85$ & 0.9996 \\
\hline 7-Azaindole & $\mathrm{y}=531,870,973.53 \mathrm{x}-232,137,687.50$ & 0.9994 \\
\hline
\end{tabular}


65 66 Table S6.
measured.

\begin{tabular}{|c|c|c|c|c|c|c|c|c|}
\hline \multirow{2}{*}{ Fuel } & \multicolumn{7}{|c|}{ Concentration (ppm) } \\
\cline { 2 - 9 } & Bin 1 & Bin 2 & Bin 3 & Bin 4 & Bin 5 & Bin 6 & Bin 7 & Total \\
\hline F-24 (1) & 0 & 1.28 & 0 & 0.74 & 7.4 & 0.56 & 0 & 9.98 \\
\hline F-24 (2) & 0 & 1.62 & 0 & 0.07 & 1.5 & 0.46 & 0 & 3.65 \\
\hline JP-5 (1) & 0 & 3.02 & 0 & 0 & 2.19 & 0.09 & 0 & 5.3 \\
\hline JP-5 (2) & 0 & 0.84 & 0 & 0.09 & 3.04 & 0 & 0 & 3.97 \\
\hline Jet A (1) & 0 & 0.14 & 0 & 0.48 & 3.93 & 1.12 & 0 & 5.67 \\
\hline Jet A (2) & 0 & 0.17 & 0 & 0.27 & 2.59 & 0.55 & 0.08 & 3.66 \\
\hline F76 (1) & 0 & 0 & 0 & 0 & 0.2 & 36.41 & 0.6 & 37.21 \\
\hline F76 (2) & 0 & 0 & 0 & 0 & 3.3 & 116.7 & 1.8 & 121.8 \\
\hline MGO (1) & 0 & 0 & 0 & 0.8 & 28.7 & 71.21 & 1.03 & 101.74 \\
\hline MGO (2) & 0 & 0 & 0 & 3.53 & 63 & 99.9 & 3.52 & 169.95 \\
\hline
\end{tabular}

67

68

69

70

71 


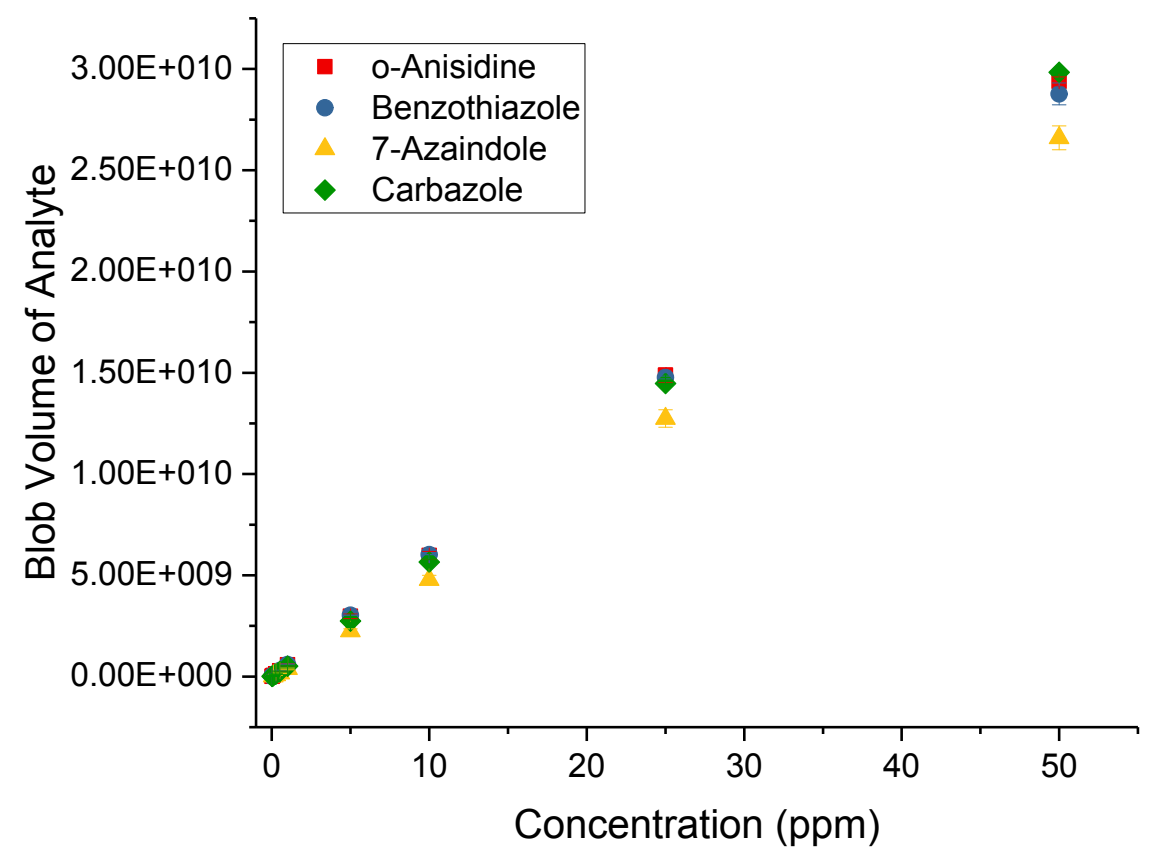

72

73 Figure S1. The blob volumes for o-ansidine, benzothiazole, 7-azaindole and carbazole were 74 plotted against the $\mathrm{ppb} / \mathrm{ppm}$ of nitrogen. The average relative standard deviation of blob volumes 75 for all standards over the entire range of concentrations was $9.10 \%$.

76 